\title{
EP-864 CIRCADIAN CLOCK EXPRESSION IN ANTERIOR PITUITARY GLAND IS ALTERED IN DIFFERENT THYROID CONDITIONS.
}

Bargi-Souza P1, Peliciari-Garcia RA'1,2, Nunes MT ${ }^{1}$ - $^{1}$ Department of Physiology and Biophysics of the Institute of Biomedical Sciences-I, University of São Paulo, SP, Brazil; ${ }^{2}$ Department of Biological Sciences, Federal University of São Paulo, Diadema, SP, Brazil - paulascj@gmail.com

\section{CONTEXT AND OBJECTIVES}

The hypo and hyperthyroidism alter the synthesis/secretion of pituitary hormones, which in normal conditions present fluctuations in serum concentration during the $24 \mathrm{~h}$ period. An intrinsic pituitary circadian clock might be related to these oscillations; however, the possible interaction between thyroid hormonal conditions and circadian clock gene expression in anterior pituitary is still unknown. The purpose of this study was to investigate the expression of core clock components as Bmal1, Per2 and Clock, as well as Tshb, Prl, Lhb and Dio2 as markers of thyrotroph, lactotroph, gonadotroph function and thyroid hormone action, respectively, during hypo and hyperthyroidism in rats.

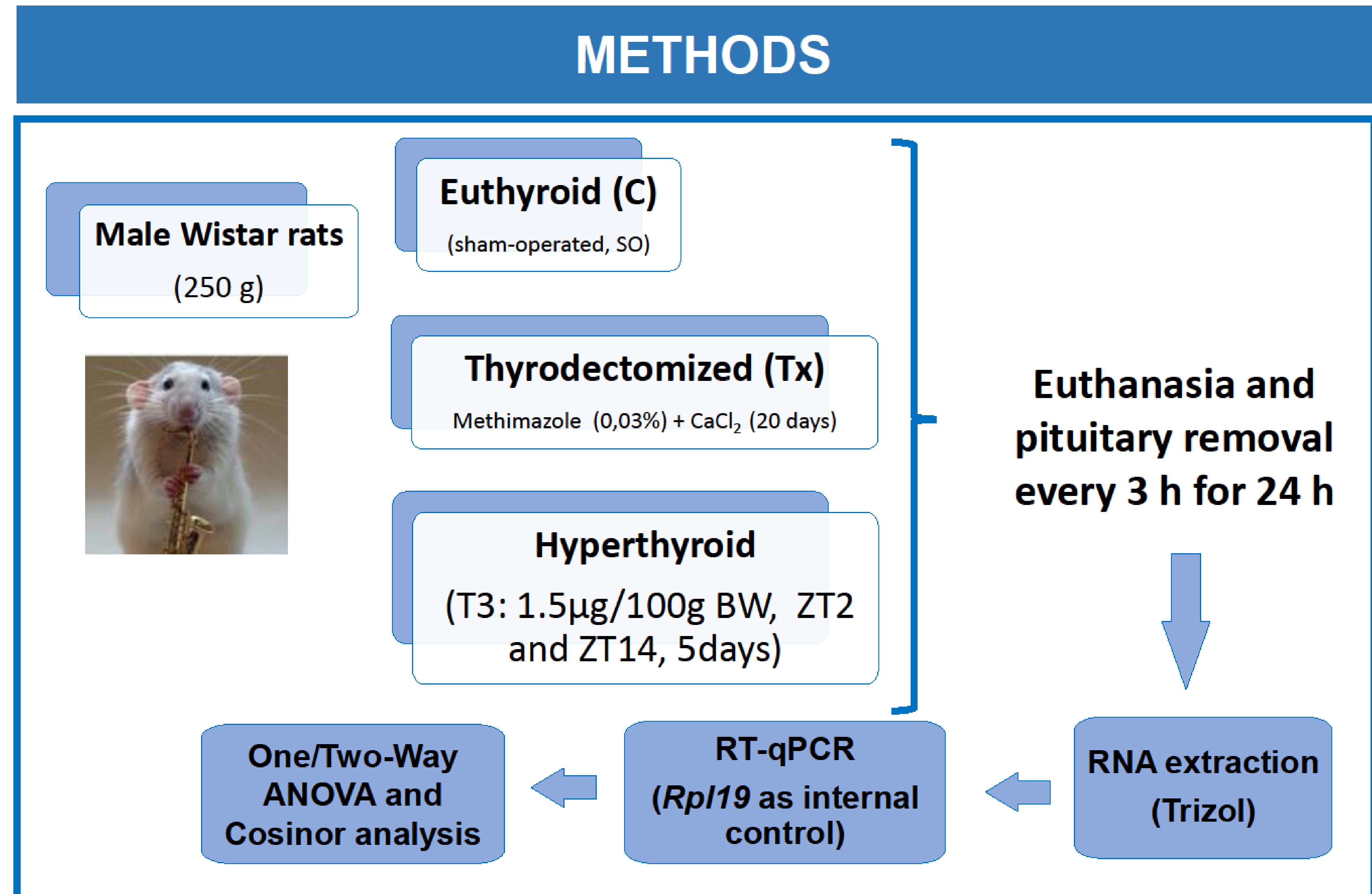

\section{RESULTS}

The expression of Bmal1, Per2, Dio2, Prl and Lhb presented a circadian rhythmicity in anterior pituitary of euthyroid rats. Per2 and Dio2 acrophases occurred around ZT 12, while Bmal1, Lhb and Prl were at ZT 0/24. In the hypo and hyperthyroid animals, the circadian expression of Per2 and Lhb was abolished. Bmal1 lost its circadian pattern of expression during hypothyroidism, while the hyperthyroidism reduced its amplitude and mesor values. Prl expression lost its circadian pattern of rhythmicity during hyperthyroidism, while the hypothyroidism reduced its amplitude and mesor values and delayed the acrophase. In hypothyroid animals the acrophase of Dio2 was advanced and mesor was higher than in euthyroid rats, while in hyperthyroidism its circadian pattern was lost.
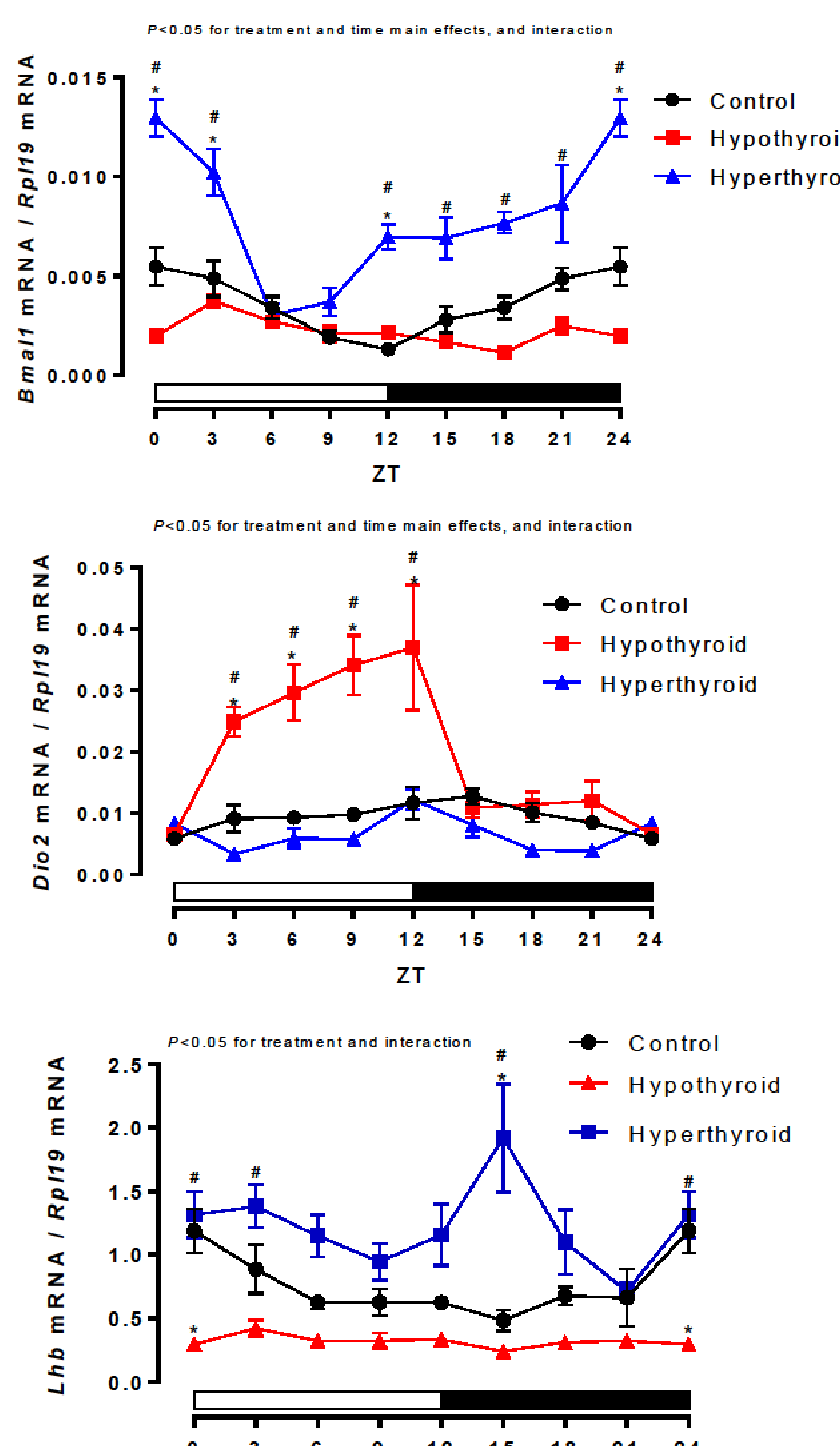

$\mathrm{ZT}$

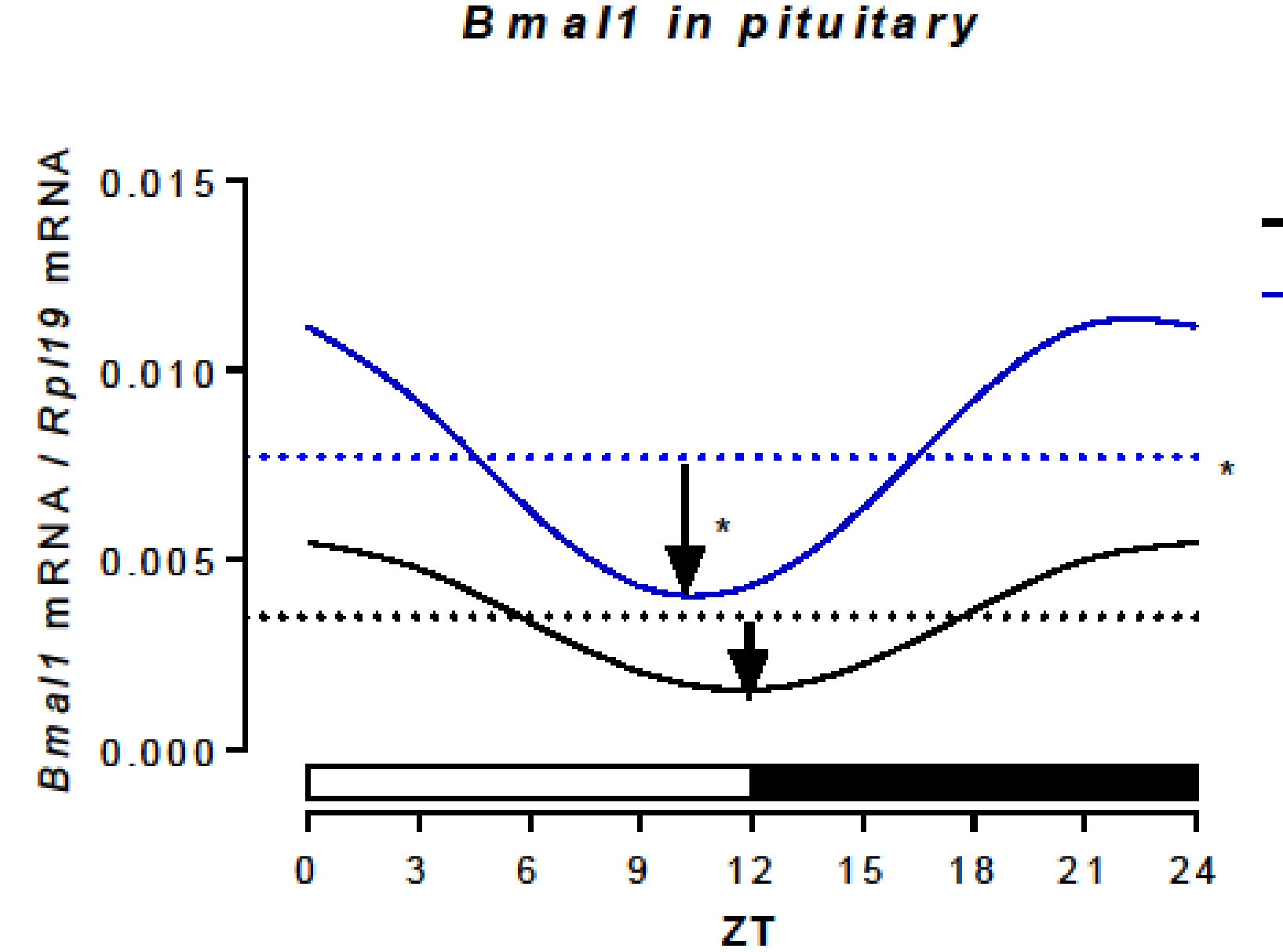

Dio 2 in pituitary

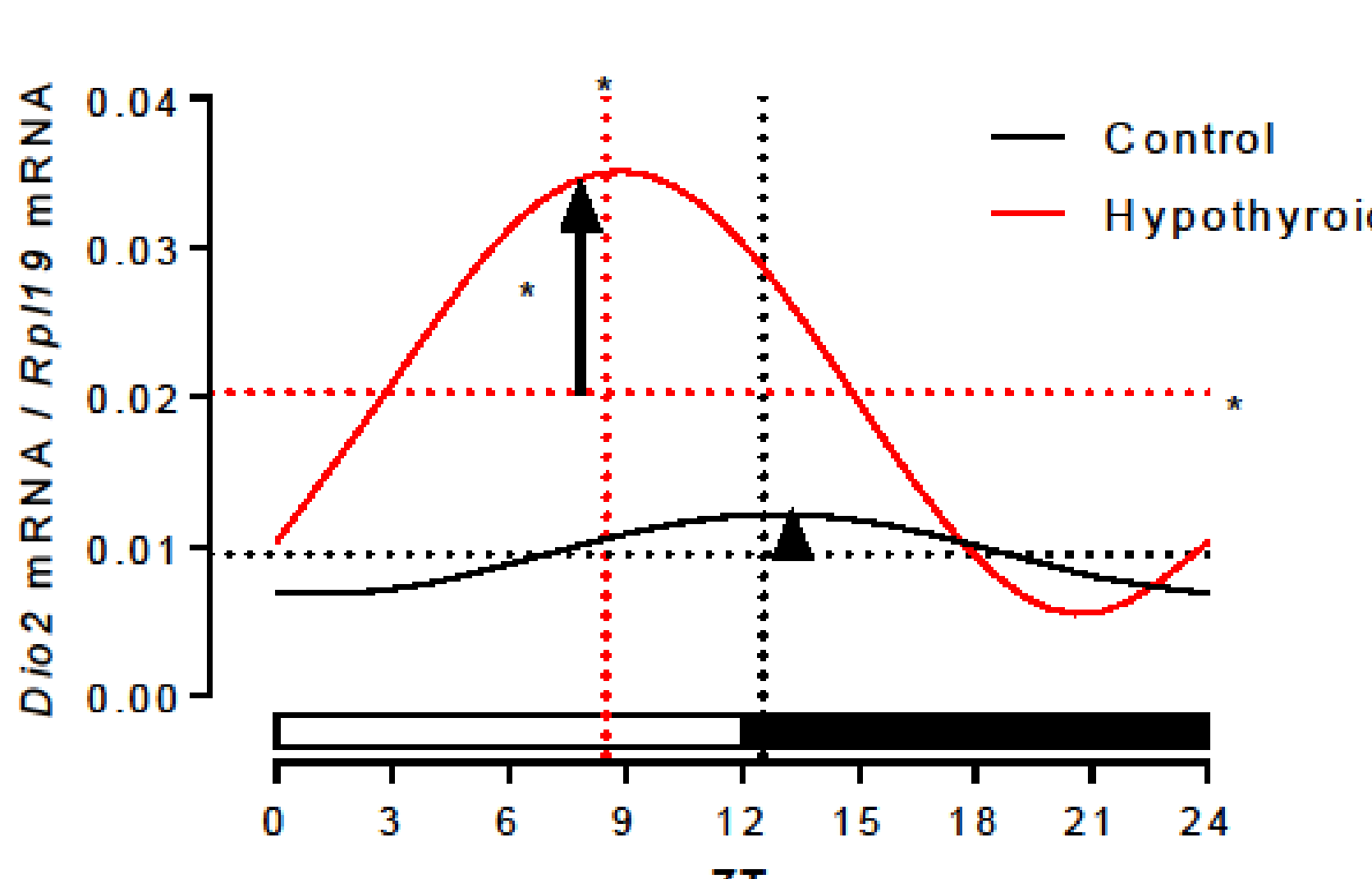

Lhb in pituitary

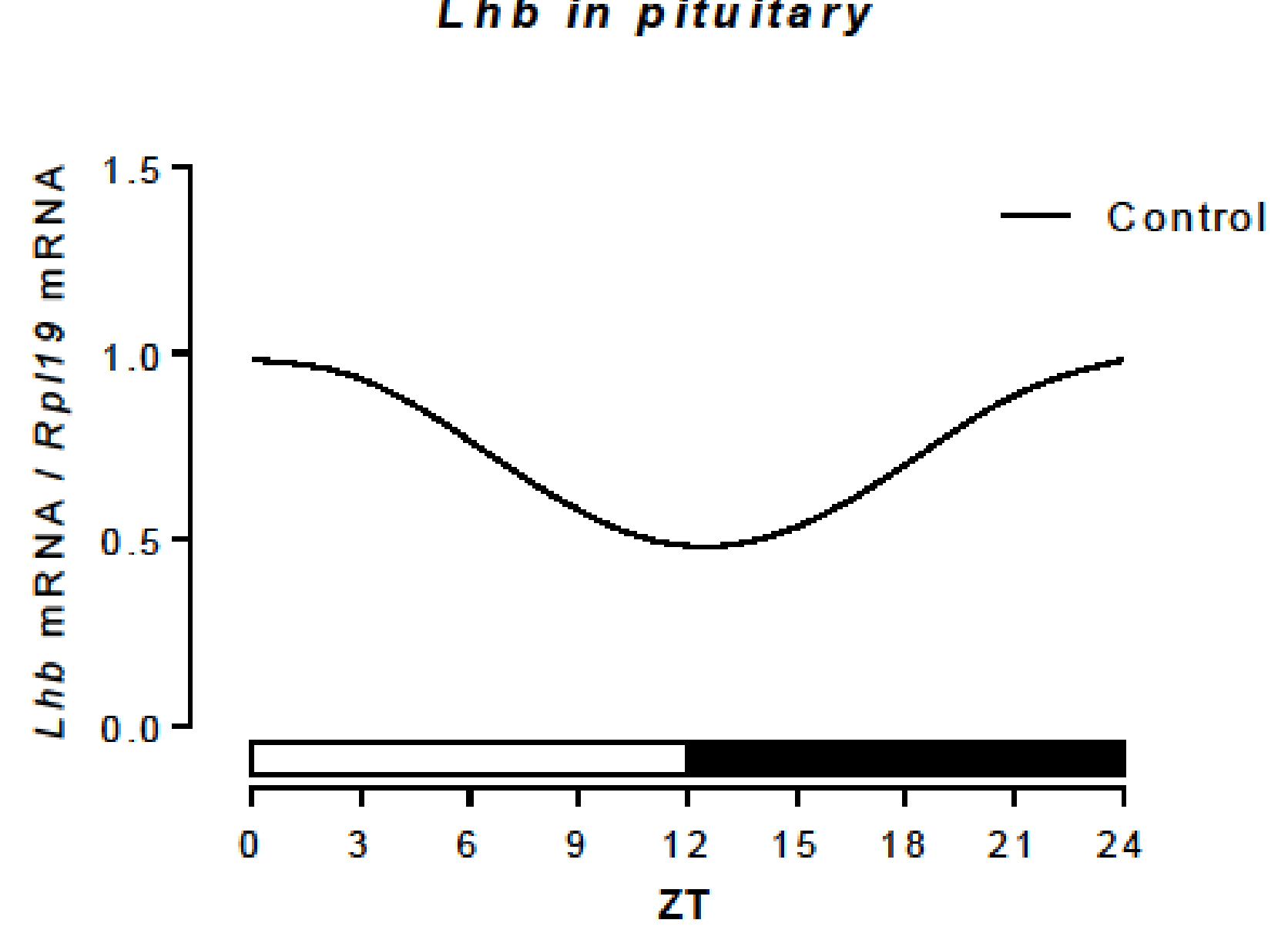

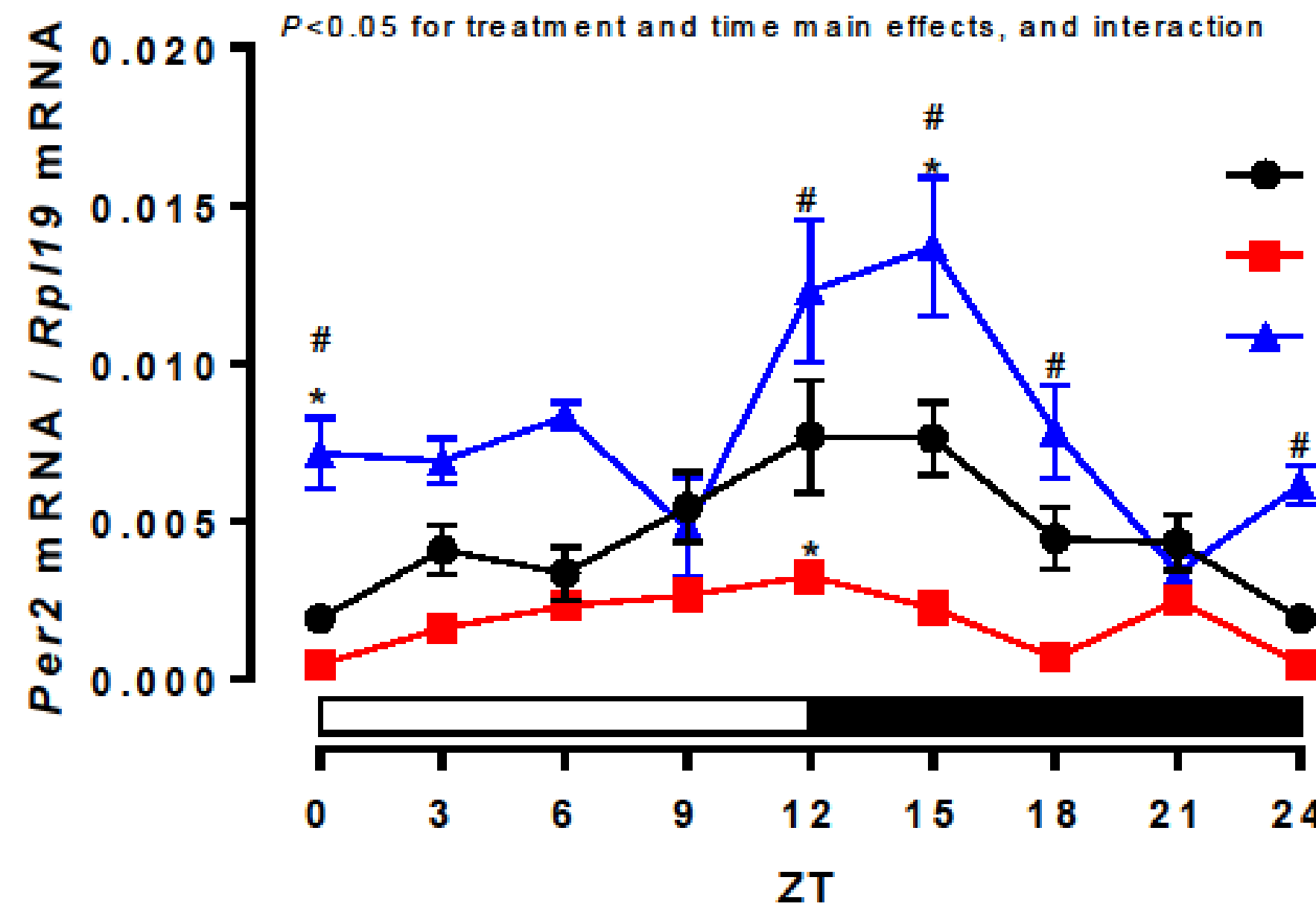
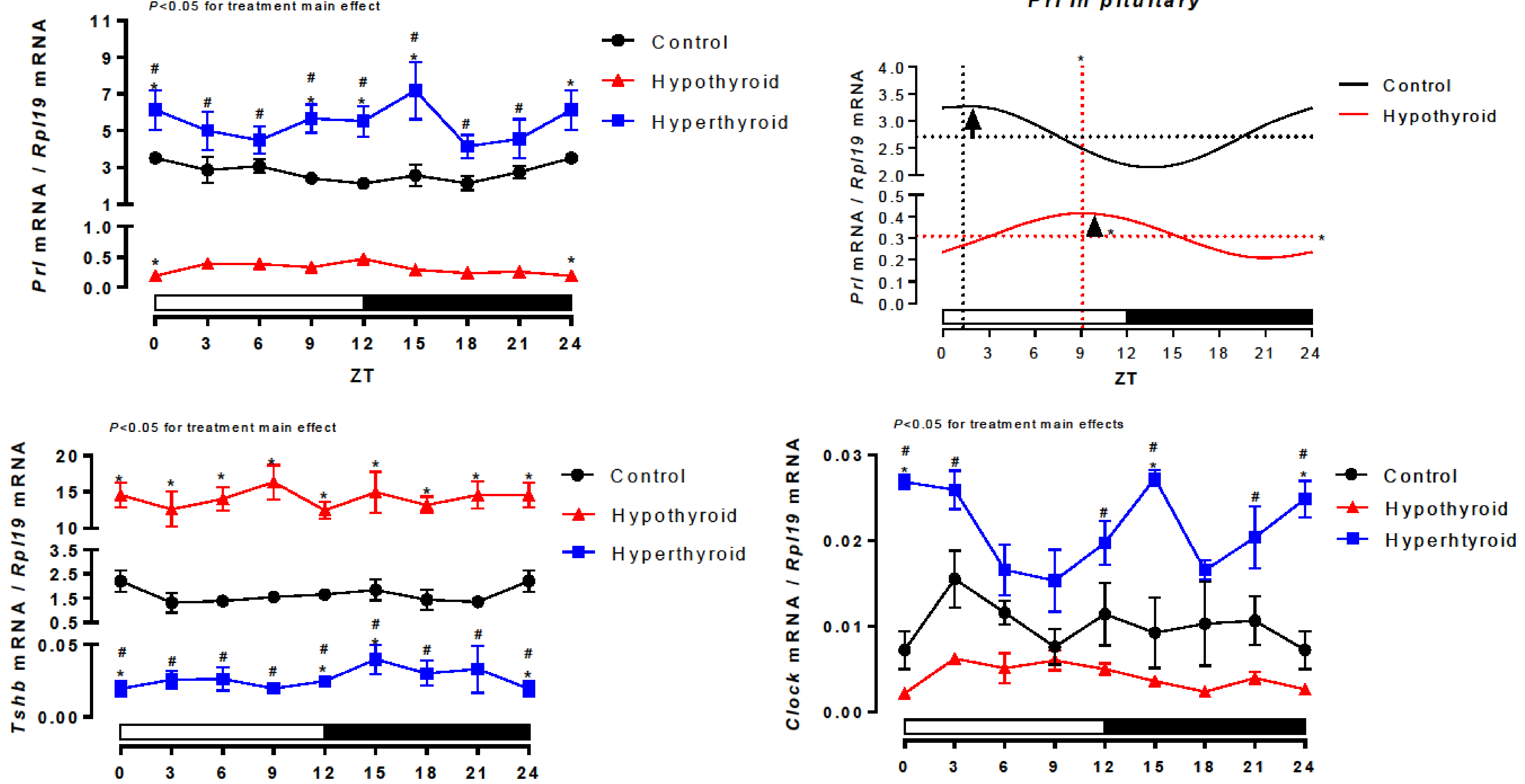

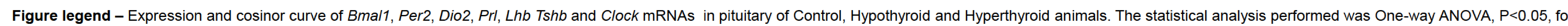
both groups in all cases, and Two-way ANOVA, ${ }^{*} P<0.05$ vs Control at respective ZTs, \# $P<0.05$ in the comparison between Hypothyroid and Hyperthyroid at respective ZTs . $n=6 / Z T / g r o u p$. $Z T=Z$ eitgeber Time

Our study reveals that the expression of core clock components in anterior pituitary gland are altered during the hyper and hypothyroidism, which might contribute to the altered secretion of pituitary hormones observed in these pathological conditions.

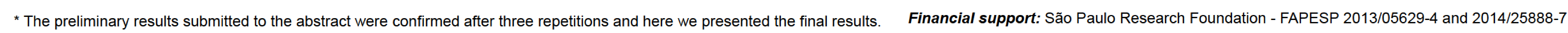

